\title{
An fMRI Investigation of Analogical Mapping in Metaphor Comprehension: The Influence of Context and Individual Cognitive Capacities on Processing Demands
}

\author{
Chantel S. Prat \\ University of Washington
}

\author{
Robert A. Mason and Marcel Adam Just \\ Carnegie Mellon University
}

\begin{abstract}
This study used fMRI to investigate the neural correlates of analogical mapping during metaphor comprehension, with a focus on dynamic configuration of neural networks with changing processing demands and individual abilities. Participants with varying vocabulary sizes and working memory capacities read 3-sentence passages ending in nominal critical utterances of the form " $\mathrm{X}$ is a $\mathrm{Y}$." Processing demands were manipulated by varying preceding contexts. Three figurative conditions manipulated difficulty by varying the extent to which preceding contexts mentioned relevant semantic features for relating the vehicle and topic of the critical utterance to one another. In the easy condition, supporting information was mentioned. In the neutral condition, no relevant information was mentioned. In the most difficult condition, opposite features were mentioned, resulting in an ironic interpretation of the critical utterance. A fourth, literal condition included context that supported a literal interpretation of the critical utterance. Activation in lateral and medial frontal regions increased with increasing contextual difficulty. Lower vocabulary readers also had greater activation across conditions in the right inferior frontal gyrus. In addition, volumetric analyses showed increased right temporo-parietal junction and superior medial frontal activation for all figurative conditions over the literal condition. The results from this experiment imply that the cortical regions are dynamically recruited in language comprehension as a function of the processing demands of a task. Individual differences in cognitive capacities were also associated with differences in recruitment and modulation of working memory and executive function regions, highlighting the overlapping computations in metaphor comprehension and general thinking and reasoning.
\end{abstract}

Keywords: metaphor, fMRI, analogical reasoning, working memory, right hemisphere

Metaphor comprehension is like analogical reasoning; both involve comparing two superficially dissimilar concepts and selecting and mapping the relevant features from one to the other. This is especially true when the metaphors are nominal in form ( $\mathrm{X}$ is a $\mathrm{Y})$. To understand the statement "Research is a diamond mine," one must first select the pertinent features of the vehicle (diamond mine) and then map them to the topic of the metaphor (research). Similarly, to understand an analogy between the process of conducting research and mining for diamonds, one needs to select the relevant features of mining for diamonds (e.g., methodical search, big payoff) and apply them to their representation of the scientific process. According to Genter and colleagues' structure mapping

This article was published Online First November 28, 2011.

Chantel S. Prat, Department of Psychology and Institute for Learning and Brain Sciences, University of Washington; Robert A. Mason and Marcel Adam Just, Department of Psychology, Carnegie Mellon University.

This work was supported by National Institute of Mental Health Grant MH029617 to Marcel Adam Just and by National Institute on Deafness and Communication Disorders Grant DC009634 to Chantel S. Prat. We would like to thank Andrea Stocco, Kara Cohen, and Jennifer Moore for their comments and contributions to this article.

Correspondence concerning this article should be addressed to Chantel S. Prat, University of Washington, Department of Psychology and Institute for Learning and Brain Sciences, Box 351525. E-mail: csprat@uw.edu theory (e.g., Gentner, 1983; Gentner, Bowdle, Wolff, \& Boronat, 2001; Gentner \& Clement, 1988), this initial semantic comparison between two superficially dissimilar items is virtually the same in metaphor and analogy, given that the metaphor is relatively novel. Much debate has occurred about the nature of this comparison and how it evolves as a metaphor moves from novel to familiar (see Glucksberg, 2003, for a discussion), but the idea that there is substantial overlap between the cognitive processes involved in metaphor comprehension and analogical reasoning is uncontroversial (Gentner et al., 2001; Gentner \& Clement, 1988; Sternberg \& Nigro, 1983; Zinken, 2007). This article capitalizes on the overlapping computations of metaphor comprehension and analogical reasoning, using the relatively well-established brain-behavior mappings in component processes of analogical reasoning as a starting point for better understanding the neural basis of metaphor comprehension.

\section{The Neural Correlates of Analogy and Metaphor}

Although the patterns of activation observed in neuroimaging investigations of metaphor comprehension and analogical reasoning are rather similar, somewhat different interpretations of the roles of the various cortical regions have been reached by researchers studying metaphor versus those studying analogy. This is particularly apparent when considering the proposed roles of the two hemispheres. It is clear that both types of tasks rely exten- 
sively on computations executed in lateral prefrontal regions (e.g., Bunge, Wendelken, Badre, \& Wagner, 2005; Cho et al., 2010; Geake \& Hansen, 2010; Krawczyk, McClelland, Donovan, Tillman, \& Maguire, 2010; Rapp, Leube, Erb, Grodd, \& Kircher, 2004; Schmidt \& Seger, 2009; Shibata, Abe, Terao, \& Miyamoto, 2007; Volle, Gilbert, Benoit, \& Burgess, 2010; Wendelken, Nakhabenko, Donohue, Carter, \& Bunge, 2008; Wright, Matlen, Baym, Ferrer, \& Bunge, 2007; Yang, Edens, Simpson, \& Krawczyk, 2009). Left lateral prefrontal regions activate consistently in both metaphor comprehension paradigms (e.g., Eviatar \& Just, 2006; Rapp et al., 2004; Rapp, Leube, Erb, Grodd, \& Kircher, 2007; Shibata et al., 2007) and in analogical reasoning paradigms (Bunge et al., 2005; Cho et al., 2010; Geake \& Hansen, 2010; Wendelken et al., 2008). Researchers investigating analogical reasoning have further subdivided the left lateral prefrontal cortex (LLPFC) into at least two functionally distinct regions, with activation in the more anterior areas (e.g., BA 10, 11, and 47) being attributed to relational integration and mapping (e.g., Bunge et al., 2005; Green, Fugelsang, Kraemer, Shamosh, \& Dunbar, 2006; Wendelken et al., 2008) and activation in the more posterior areas (e.g., BA 44 and 45) being attributed to semantic analysis in verbal analogies (e.g., Bunge et al., 2005). Corresponding regions of the right lateral prefrontal cortex (RLPFC) seem to be sensitive to the processing demands of the task (e.g., Bunge, Helskog, \& Wendelken, 2009; Bunge et al., 2005) and may serve a more general role in response selection or goal-directed behavior (Aron, Robbins, \& Poldrack, 2004; Bunge et al., 2005, 2009; Dodds, MoreinZamir, \& Robbins, 2010; Goghari \& MacDonald, 2009).

Research on the neural correlates of metaphor comprehension, on the other hand, has historically focused on the role of right hemisphere (RH) contributions. Early neuropsychological research suggested that the RH was necessary for understanding figurative speech (Brownell, Simpson, Bihrle, Potter, \& Gardner, 1990; Rinaldi, Marangolo, \& Baldassarri, 2004), and some neuroimaging research has supported this claim (Mashal, Faust, Hendler, \& Jung-Beeman, 2007; Stringaris et al., 2006). However, another series of neuroimaging studies found only left hemisphere (LH) involvement in metaphor comprehension (Lee \& Dapretto, 2006; Rapp et al., 2004, 2007; Shibata et al., 2007; Stringaris, Medford, Giampietro, Brammer, \& David, 2007), and a recent meta-analysis of neuroimaging investigations of discourse found no evidence of $\mathrm{RH}$ contributions to metaphor processing that were unaccompanied by LH contributions (Ferstl, Neumann, Bogler, \& von Cramon, 2008). These results seem to indicate that not all metaphor processing requires unique RH contributions. What remains unclear is how and when the RH may become involved in metaphor comprehension. Existing theories of the role of the RH in complex cognitive tasks in general, and language comprehension specifically, offer several possible hypotheses about the nature and timing of RH contributions to metaphor comprehension.

Discourse comprehension research has offered several suggestions about the role of the RH in metaphor processing. According to the coarse-coding theory, the RHs centrality to metaphor comprehension arises because of its unique, coarse semantic representations (e.g., Beeman \& Chiarello, 1998; Beeman et al., 1994; Jung-Beeman, 2005; Mashal et al., 2007). This theory proposes that the RH is centrally involved in novel metaphor comprehension when the items being compared are distantly semantically related, and semantic fields in the $\mathrm{RH}$ are more likely to contain overlap- ping information from the two items. With respect to the component computations, Jung-Beeman and colleagues proposed that semantic activation, selection, and integration all occur bilaterally, in distinct cortical regions, with the lateral prefrontal regions (e.g., BA 44/45, or Broca's Area and RH homologue) being primarily involved in selection processes.

Yet another class of theories suggests that the RH supports metaphor comprehension through social and pragmatic processes such as theory of mind (Mason \& Just, 2009, 2011; Saxe \& Kanwisher, 2003; Saxe \& Powell, 2006; Saxe \& Wexler, 2005; but see also Mitchell, 2008, for a contrasting viewpoint). According to these views, the RH (especially the posterior superior temporoparietal junction) would be particularly important for metaphor processes when reasoning about the intent of the speaker (or writer) of the metaphor becomes relevant for comprehending its meaning.

A third hypothesis about the role of the $\mathrm{RH}$ in metaphor processes is that it serves as a resource reserve, providing additional albeit less efficient processing resources when the task demands are larger than what the LH can supply. The observed phenomenon is manifested as a "spillover" of the excess processing and the activation it engenders from the LH into RH homologues (Prat \& Just, 2008; Prat \& Just, 2011; Prat, Mason, \& Just, 2011). According to this dynamic spillover account, the extent to which the RH is involved in metaphor comprehension will vary depending on the amount of demand placed by the task relative to the language capacity or skill level of the individual. Support for this theory comes from two types of evidence. First, a large number of studies have reported contralateral spillover in a variety of tasks from simple letter identification (Hatta, 1982; Haun, 1981) to sentence comprehension with varying syntactic complexity (Just, Carpenter, Keller, Eddy, \& Thulborn, 1996; see also Mitchell \& Ross, 2008, for an example of spillover into LH with increasing difficulty of RH-dominant prosodic processes). Second, studies of individual differences in language comprehension show more RH involvement in less-skilled readers in a variety of language comprehension paradigms (Prat, Long, \& Baynes, 2007; Prat, Mason, \& Just, 2010; Prat et al., 2011).

The findings from the analogical reasoning literature are particularly consistent with the spillover account, suggesting that the RLPFC involvement in analogical processing is related to the amount of task demand (e.g., Bunge et al., 2005, 2009). Although these various theoretical frameworks are not mutually exclusive, they take different perspectives and make somewhat different predictions about how and when the RH becomes involved in metaphor comprehension. These predictions and their implications for understanding the neural correlates of metaphor comprehension processes are explored herein.

\section{Characterization of Processing Demands in Metaphor Comprehension}

Metaphor processing, like all complex cognitive tasks, is dynamic in nature. To accurately characterize the neural correlates of metaphor comprehension, one must be able to relate the changes in information processing demands to variations in behavior and brain function. The goal of this experiment was to investigate the brain basis of nominal metaphor comprehension, with an emphasis 
on the role of the RH, using a context manipulation to systematically vary the processing demands of the task.

One complication in assessing the neural underpinnings of metaphor comprehension is that a broad array of linguistic phenomena has been studied under the general category of metaphor. It is clear, however, that metaphor-comprehension tasks differ along several axes with important implications for both cognitive and neural processes. One such axis that has been well studied is familiarity (Mashal \& Faust, 2009; Mashal et al., 2007; Schmidt \& Seger, 2009; Yang et al., 2009). Although initially a controversial idea, researchers have convincingly shown that the literal/ figurative dimension of a statement is not as important as the frequency or familiarity of a particular interpretation of the statement (e.g., Giora, 1999; Giora, 2007). For example, the meanings of familiar (or frozen) metaphors, such as "bright student," become lexicalized and are accessed directly, even before the literal interpretation of the statement (e.g., Bowdle \& Gentner, 2005). Thus, the computational demands involved in semantic feature selection and relational mapping are only relevant for relatively novel metaphor comprehension. Difficulty is another facet of metaphor processing that has been proposed to influence comprehension. For example, Monetta, Ouellet-Plamondon, and Joanette (2006) added a secondary task to increase the complexity of a metaphor comprehension paradigm and found that healthy controls performed like RH-damaged patients. These results and the results of others suggest that variations in the general processing demands of metaphor comprehension paradigms result in changes in behavior (Monetta et al., 2006) and brain (Schmidt \& Seger, 2009; Yang et al., 2009). In the current experiment, comprehension of metaphors that are moderately familiar, but not frozen (e.g., "He is a giraffe"), was investigated.

Interpreting research on the factors related to metaphor comprehension can be complicated by the fact that it is difficult to orthogonally manipulate the relevant variables. For instance, difficulty is typically manipulated by varying either familiarity (e.g., Yang et al., 2009) or semantic distance (e.g., Schmidt \& Seger, 2009). Increased RH activation in either of these manipulations can be explained both by the dynamic spillover hypothesis (increased difficulty) and by coarse coding (increased semantic distance), as the meaning of familiar metaphors can be accessed directly.

In this experiment, difficulty and figurativeness were manipulated separately from familiarity and semantic relatedness by varying the preceding contexts in which metaphors occur. Behavioral research has shown that preceding contexts mentioning relevant properties for semantic mapping can dramatically facilitate metaphor comprehension (e.g., Glucksberg, 2003). Experimental passages were designed such that the same critical utterance of nominal form ( $\mathrm{X}$ is a $\mathrm{Y}$ ) could be interpreted in one of three ways: as a literal statement, as a sincere metaphoric statement, or as an ironic metaphoric statement. All critical utterances contained relatively familiar (not frozen), easy to understand statements that were most likely to be interpreted as a metaphor in isolation (e.g., "She is a lamb"). Thus, difficulty was manipulated by the extent to which context supported the default interpretation of the utterance.

In the easiest, supporting condition, critical utterances were preceded by a context sentence mentioning the relevant semantic feature (e.g., kind/gentle) for the most likely, metaphoric interpretation. In the most difficult, opposite context condition, the critical utterance was preceded by a context sentence mentioning a characteristic that was opposite to the relevant semantic feature (e.g., mean/harsh). Thus, in the opposite condition, context ultimately biased an ironic interpretation of the critical utterance. This condition was more difficult, because readers needed to both compute the metaphoric overlap and to interpret the intention of the speaker to be ironic/sarcastic. In a third, neutral condition of intermediate difficulty, the critical utterance was preceded by a tangential context sentence that did not mention any relevant features of the topic for understanding the metaphor. Thus, in the neutral conditions, readers needed to rely on existing knowledge to compute the relevant semantic overlap between topic and vehicle. Although the critical utterance was technically ambiguous, a behavioral norming study suggested that most readers ultimately adopted a sincere metaphoric interpretation of the critical utterance in the neutral condition, based presumably on the fact that the statements were more likely to be metaphoric than literal when presented in isolation. An additional, literal condition was included to control for figurativeness of the critical utterances. In the literal condition, the preceding context sentence set up a condition in which the critical utterance was interpreted as a literally true statement (e.g., a context sentence about watching a friend in a play about shepherds, followed by the statement, "She's a lamb.").

\section{Individual Differences in Metaphor Comprehension Ability}

The abstraction of commonalities between distinct representations is central for learning (Gentner \& Holyoak, 1997; Holyoak \& Thagard, 1997) and has thus been generally accepted as one of the key components of intelligent behavior (e.g., Sternberg, 1977). Although metaphor comprehension relies on this abstraction, relatively little research has investigated individual differences in metaphor comprehension abilities. Those studies that have been conducted, however, suggest that more intelligent individuals (Kazmerski, Blasko, \& Dessalegn, 2003), individuals with better verbal abilities (Blasko, 1999), and individuals with better analogical reasoning abilities (Trick \& Katz, 1986) all exhibit more enriched comprehension of metaphors and seem to be more sensitive to the precise relational relevance between topic and vehicle (e.g., Trick \& Katz, 1986).

Investigations of the neural basis of individual differences in metaphor comprehension have important implications both for understanding the neural correlates of metaphor processing and for discerning the nature of general intellectual abilities. For example, the dynamic RH spillover theory makes important prediction about individual differences in $\mathrm{RH}$ contributions to metaphor comprehension. According to this theory, variables related to an individual's experienced processing demands also influence the extent to which that person relies upon RH contributions (e.g., Prat, Long, \& Baynes, 2007; Prat et al., 2011). In this experiment, we investigate the neural basis of individual differences in specific linguistic abilities (vocabulary size) and general cognitive abilities (working memory capacity) during metaphor comprehension under a variety of contextually manipulated processing demands. 


\section{Method}

\section{Participants}

Data were collected from 43 undergraduate students recruited through Carnegie Mellon University. Nineteen participants were excluded because of excessive head motion (greater than $2 \mathrm{~mm}$ ). ${ }^{1}$ The 24 remaining participants were all right-handed, native English speakers (11 men, 13 women). All individuals gave informed consent and were paid for their participation.

\section{Materials}

The stimulus set was composed of 46 three-sentence passages. Forty of these were experimental passages, and six were filler stories that were interspersed with the experimental passages and were followed by yes or no comprehension questions to ensure that participants were reading and understanding texts. The 40 experimental passages were made up of 10 passages in each of the four context conditions: Supporting, Neutral, Opposite, and Literal. The experimental passages were designed such that the first and final sentences were identical across conditions. The second sentence was manipulated across conditions to provide varied context, biasing either a sincere metaphoric, ironic, or literal interpretation of the final sentence (or not biasing any interpretation in the neutral condition). The first sentence of each passage introduced the main character (or characters). The final sentence of each passage involved a nominal utterance of the form ( $\mathrm{X}$ is a $\mathrm{Y}$ ) made by one of the characters. Sample passages are listed in Table 1.

Four versions of the task were created such that all passages occurred in all conditions across lists. Thus, each participant saw the critical utterances in one of the four context conditions. Passages in the four conditions were equated for average length and complexity. In addition, a norming study was conducted with Carnegie Mellon undergraduates to ensure that all passages were equated for familiarity, were comprehensible, and were interpreted in the manner that they were intended to be along axes of literalness (literal vs. figurative) and sincerity (sincere vs. sarcastic). They were also checked to confirm that contextual manipulations evoked the appropriate reactions. Supporting, Neutral, and Opposite passages were all rated as figurative and thus understood to be metaphorical in nature, whereas the Literal passages were rated as literal. Both Supporting and Literal passages were rated as sincere, whereas Opposite passages were rated as sarcastic. Neutral pas-

Table 1

Sample Stimuli

\begin{tabular}{|c|c|}
\hline Sentence description & Text \\
\hline Sentence 1: Introduction & $\begin{array}{l}\text { Samantha was the first of her friends to get } \\
\text { married. }\end{array}$ \\
\hline \multicolumn{2}{|l|}{ Sentence 2: Context } \\
\hline Supporting context & Her husband anticipated her every need. \\
\hline Neutral context & She met her husband in high school. \\
\hline Opposite context & Her husband never helped around the house. \\
\hline Literal Context & $\begin{array}{l}\text { Everyone was excited about her husband's } \\
\text { royal bloodlines. }\end{array}$ \\
\hline $\begin{array}{l}\text { Sentence 3: Critical } \\
\text { utterance }\end{array}$ & She said "He is a prince." \\
\hline
\end{tabular}

sages were mostly rated as sincere but were more variable, as predicted, because of the lack of content information.

To assess the relative difficulty of comprehension of the various passages, we conducted a behavioral norming study in which each passage was followed by a comprehension question probing the reader's interpretation of the critical utterance. Reading times were collected for the context sentences, critical utterances, and comprehension questions. As expected, there were no differences among conditions in the reading times for the context sentences $(p>.10)$. Reading times did differ significantly for the critical utterances, $F(3,11)=13.53, p<.001$; these reading times were reliably fastest in the supporting condition and slowest in the opposite condition, with literal and neutral conditions falling between. Reading times for the question also differed significantly among conditions, $F(3,11)=6.71, p=.001$. Reading times for critical utterances in the four conditions are depicted in Figure 1.

The experimental stimuli were created specifically for this experiment. The filler passages were a modified subset of passages used in Eviatar and Just (2006).

\section{Procedure}

All participants underwent behavioral testing and fMRI practice sessions 1 or 2 days prior to their scan. During the practice sessions, the Reading Span Test (Daneman \& Carpenter, 1980), the Nelson-Denny Reading Test (Brown, Fishco, \& Hanna, 1993), and the Edinburgh Handedness Inventory (Oldfield, 1971) were administered first, in that order. Then each participant practiced the paradigm with sample stimuli inside a mock scanner.

During the experimental fMRI scan, passages were rearprojected onto a screen that participants viewed through a mirror attached to the head coil at a visual angle of approximately $30^{\circ}$.

Four 30-s fixations occurred: one at the beginning, two evenly spaced throughout the experiment, and one at the end. During these fixations, participants viewed an " $\mathrm{X}$ " in the center of the screen and were told to relax and clear their mind. Participants were instructed to read the passages for comprehension and were told that they would sometimes (randomly) be required to answer a question following a passage. The first and second sentences of each experimental passage appeared simultaneously on the screen and remained visible for $9 \mathrm{~s}$. Afterward, an " $\mathrm{X}$ " was displayed on the screen for $4 \mathrm{~s}$, followed by the critical utterance, which appeared for $4 \mathrm{~s}$. After each passage, another " $\mathrm{X}$ " appeared in the center of the screen for a 7-s rest period. In filler passages, a question appeared on the screen for $4 \mathrm{~s}$ after the completion of the 7 -s rest. Participants were instructed to press a mouse button corresponding to either "yes" or "no" in response to the comprehension question. Another 7-s rest, with an " $X$ " on the screen, followed each comprehension question.

\footnotetext{
${ }^{1}$ Thirteen of the 19 participants who were excluded for motion were also run on a relatively demanding dual-task paradigm before completing this experiment. Thus, this relatively low yield may have arisen because some participants were experiencing mental fatigue. Thirteen of the participants with useable data completed this experiment first, whereas of them completed another experiment before this one.
} 


\section{Reading Times to Critical Utterances as a function of Context Condition}

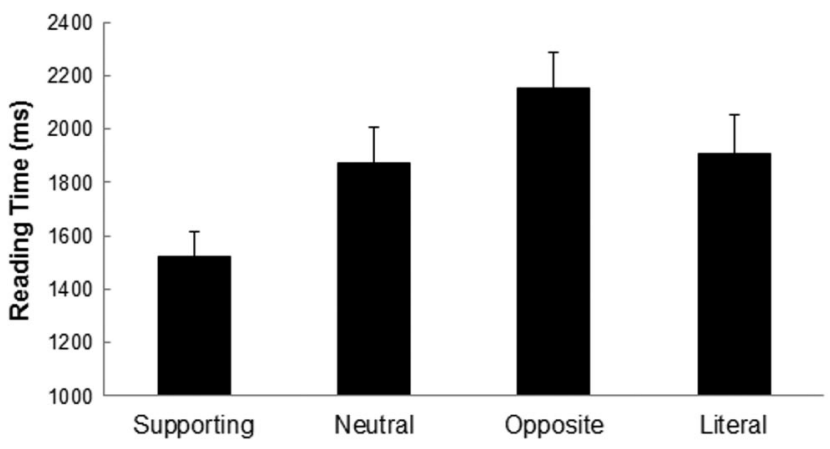

Figure 1. Bar graphs depicting the mean reading times to critical utterances as a function of the four preceding context conditions (error bars depict standard error of the mean) from the behavioral norming investigation.

\section{fMRI Data Acquisition}

The data were collected using a Siemens Allegra 3.0T scanner at the Brain Imaging Research Center of the University of Pittsburgh Medical Center, jointly established by Carnegie Mellon University and the University of Pittsburgh. The study was performed with a gradient echo planar pulse sequence with TR = $1,000 \mathrm{~ms}, \mathrm{TE}=30 \mathrm{~ms}$, and a $60^{\circ}$ flip angle. Seventeen obliqueaxial slices were imaged, and each slice was $5 \mathrm{~mm}$ thick with a gap of $1 \mathrm{~mm}$ between slices. The acquisition matrix was $64 \times 64$ with $3.125-\mathrm{mm} \times 3.125-\mathrm{mm} \times 5-\mathrm{mm}$ voxels.

\section{Distribution of Activation Analyses}

The data were analyzed using SPM2 (Wellcome Department of Imaging Neuroscience, www.fil.ion.ucl.ac.uk/spm) to examine the distribution of activation during critical utterance comprehension as a function of preceding context and individual vocabulary size and working memory capacity. Images were corrected for slice acquisition timing, motion-corrected, normalized to the Montreal Neurological Institute (MNI) template, resampled to $2 \times 2 \times 2$ $\mathrm{mm}$ voxels, and smoothed with an $8-\mathrm{mm}$ Gaussian kernel to decrease spatial noise. Statistical analyses were performed on individual and group data using the general linear model as implemented in SPM2 (Fritson et al., 1995). Only results falling within gray matter were reported. For individual participants, a fixed-effects model that incorporated a high-pass filter with a cutoff of $378 \mathrm{~s}$ and an AR(1) correction for serial autocorrelation was used to estimate parameters. The model included one regressor for the critical utterances in each of the four experimental conditions and one regressor for all of the context sentences averaged together. These regressors were boxcar functions spanning the interval between stimulus onset and offset, convolved with the hemodynamic response. Group analyses were performed using a random-effects model.

Context effects in the three difficulty conditions were calculated by computing the contrast between the critical utterances and the averaged contexts across conditions. Context sentences, rather than the literal passages, were used as a linguistic baseline, be- cause the literal passages were relatively difficult to understand (see Figure 1). The unexpected difficulty of the literal sentences arose because their predications (e.g., "She [here referring to a female young sheep] is a lamb") were strange and tended to be interpreted metaphorically. Reaction time data and activation maps suggested that despite having contexts that supported the literal interpretation (e.g., watching a play about shepherds), readers had difficulty comprehending the critical utterances in literal contexts. Thus, context sentences were averaged across the four conditions to control for any differences in context that might arise because of predictive inferences that could potentially be drawn in the supporting or opposite conditions but not in the neutral or literal conditions. The resulting averaged context created the same linguistic baseline for each of the four critical utterance conditions.

Figurativeness effects were calculated both by subtracting each of the figurative conditions from the literal condition and by subtracting the average of the three figurative conditions from the literal condition. False discovery rate (FDR) corrections (Genovese, Lazar, \& Nichols, 2002) were applied to the group analyses with a corrected height threshold of $p<.05$ and an extent threshold of 12 voxels, roughly corresponding to the volume of two voxels in native space.

Individual differences in critical utterance comprehension were assessed on a voxel-wise basis using random-effects multiple regression models in which Nelson-Denny Vocabulary percentile scores and Reading Span Scores were entered simultaneously as independent variables and the contrast parameter estimates of interest served as the dependent variables. Nelson-Denny Vocabulary percentiles ranged from 38-97 $(M=82.5, S D=13.6)$. Reading Span scores ranged from 2.0 to $5.0(M=3.27, S D=$ 0.90). Nelson-Denny Vocabulary and Reading Span were moderately positively correlated, $r(24)=.30$, although this correlation was not significant ( $p=.16$ ). An uncorrected height threshold of $p<.001$ and an extent of 12 voxels were used for individual differences analyses.

\section{Volumetric Analyses}

Nine functionally defined regions of interest (ROIs) were selected from a larger group of 21 spherical ROIs defined a priori to encompass all of the major regions of activation across five discourse-comprehension experiments conducted at the Center for Cognitive Brain Imaging (also used in Prat et al., 2011). We chose ROIs that have been reported to be important in metaphoric and analogical processes, including five lateral frontal ROIs (three in the $\mathrm{LH}$ and two in the $\mathrm{RH}$ ) and two medial frontal ROIs, as well as ROIs that are important for discourse comprehension (bilateral superior/posterior temporal regions corresponding to Wernicke's area and its RH homologue). In this set of ROIs, the majority of RH ROIs defined were slightly larger than those in the LH because of greater variability between participants in activation foci in the RH (see Prat et al., 2011). MNI coordinates, Brodmann's areas, and sphere radii for each of the nine ROIs are listed in Table 2.

The number of voxels activated in each ROI above the height threshold of $p<.05$, FDR corrected, was calculated for each participant independently for each of the three critical utterance versus context contrasts. The effects of difficulty for each ROI were analyzed separately using one-way analyses of variance (ANOVAs), with the three levels of difficulty as within-subject 
Table 2

Centroids, Size, and Brodmann's Areas for Nine Regions of Interest Used in Volumetric Analyses

\begin{tabular}{|c|c|c|c|c|c|}
\hline \multirow[b]{2}{*}{ Cortical region } & \multirow{2}{*}{$\begin{array}{l}\text { Brodmann's area } \\
\text { for centroid }\end{array}$} & \multirow[b]{2}{*}{ Radius } & \multicolumn{3}{|c|}{ Centroid MNI coordinates } \\
\hline & & & $x$ & $y$ & $z$ \\
\hline LH Inferior/Orbital Frontal & 47 & 12 & -48 & 30 & -10 \\
\hline LH Inferior/Middle Frontal & 45 & 14 & -48 & 18 & 18 \\
\hline LH Superior Frontal & 6 & 14 & -40 & 2 & 52 \\
\hline Inferior/Anterior Medial Frontal & 9 & 14 & -6 & 56 & 40 \\
\hline Superior/Posterior Medial Frontal & 6 & 14 & -2 & 10 & 50 \\
\hline RH Inferior/Orbital Frontal & 47 & 14 & 52 & 30 & -14 \\
\hline RH Inferior/Middle Frontal & 46 & 14 & 48 & 22 & 26 \\
\hline LH Superior Posterior Temporal & 40 & 14 & -52 & -54 & 18 \\
\hline RH Temporo-Parietal Junction & $22 / 39$ & 18 & 48 & -50 & 6 \\
\hline
\end{tabular}

Note. $\quad \mathrm{MNI}=$ Montreal Neurological Institute; $\mathrm{LH}=$ left hemisphere; $\mathrm{RH}=$ right hemisphere.

variables. The effect of figurativeness was calculated independently for each ROI using a $t$ test comparing the number of active voxels in the literal condition with the average number of voxels across the three figurative conditions. All effects were tested at a significance level of $p<.05$.

\section{Results}

\section{Effects of Difficulty}

Group distribution of activation analyses showed that increased difficulty was associated with increased activation in a bilaterally distributed language comprehension network. The amount of neural resources devoted to processing identical critical utterances varied dramatically as a function of the extent to which the preceding context facilitated the default, sincere metaphor interpretation. In the easiest condition, when critical utterances were preceded by supporting contexts, increased activation was observed only in the left inferior orbital frontal region and in the hippocampus. In the most difficult condition, when critical utterances were preceded by opposite contexts, increased activation was observed in a large, bilateral network, including bilateral and medial frontal areas, bilateral temporal areas, bilateral parietal regions, and in memory regions, including hippocampus and precuneus. Levels of activation in the neutral condition, when no relevant context was given, fell in between the easy and difficult conditions, with increased activation throughout the left frontal regions (including inferior, middle, and superior frontal gyri), as well as in left temporal and parietal regions and in memory regions (including hippocampus and precuneus). The contrast of the difficult (opposite) minus the easy (supporting) conditions revealed reliably greater activation in bilateral lateral frontal and medial frontal regions, as well as in the left temporal lobe and in the striatum. The direct contrasts of difficult minus medium (neutral) and medium minus easy did not reach significance. There were no regions in which easier conditions resulted in more activation than more difficult ones. Activation maps depicting these context effects (activation in critical figurative utterances preceded by the three types of context) and the direct subtraction of difficult minus easy conditions are depicted in Figure 2. MNI

coordinates, Brodmann's areas, and peak T values of clusters of activation in each of the three conditions, and in the direct subtraction of difficult minus easy conditions, are listed Table 3.

Volumetric analyses provided additional evidence that difficulty influenced the comprehension of critical utterances. As in the distribution of activation results, the extent to which context supported a sincere metaphoric interpretation was inversely proportionate to the amount of activation observed in several bilateral frontal regions. Reliable effects of context difficulty were found in the three left lateral frontal ROIs, in the anterior, inferior medial frontal ROI, and in the two right lateral frontal ROIs. ANOVA statistics, mean voxel counts, and follow-up

\section{Distribution of Activation Maps of the Influence of Contextual Support on Metaphor Comprehension}

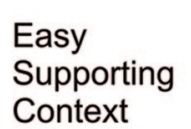
Medium
Neutral
Context

\section{Difficult Opposite Context}
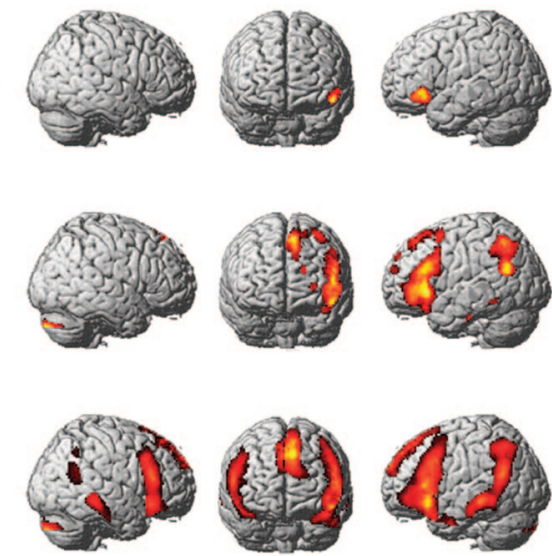

\section{Difficult > Easy}
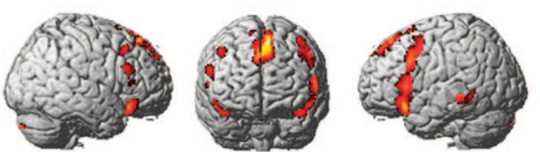

Figure 2. Activation maps showing distribution of activation analyses for critical utterances in each of the three context conditions and for the contrast of the difficult minus easy context conditions (bottom). 
Table 3

Significant Clusters of Activation in the Three Critical Utterance Conditions Minus All Context, and in the Difficult Minus Easy Contrast

\begin{tabular}{|c|c|c|c|c|c|c|}
\hline \multirow[b]{2}{*}{ Cortical region } & \multirow[b]{2}{*}{ Peak Brodmann's area } & \multirow[b]{2}{*}{ Cluster size } & \multirow[b]{2}{*}{ Peak $\mathrm{T}$ value } & \multicolumn{3}{|c|}{ MNI coordinates } \\
\hline & & & & $x$ & $y$ & $z$ \\
\hline \multicolumn{7}{|l|}{ (A) Easy: Supporting condition } \\
\hline Left Inferior Orbital Frontal & 47 & 326 & 5.93 & -46 & 32 & -4 \\
\hline Hippocampus & & 169 & 6.36 & -24 & -48 & 8 \\
\hline \multicolumn{7}{|l|}{ (B) Medium: Neutral condition } \\
\hline Left Inferior Frontal & 45 & 2,755 & 5.57 & -50 & 28 & 8 \\
\hline Left Superior Medial Frontal & 8 & 747 & 6.20 & -8 & 34 & 48 \\
\hline Left Frontopolar & 10 & 53 & 4.29 & -18 & 58 & 22 \\
\hline Left Frontopolar & 10 & 61 & 4.11 & -28 & 46 & 4 \\
\hline Left Superior Medial Frontal & 9 & 63 & 3.77 & -8 & 56 & 38 \\
\hline Left Middle Frontal & 6 & 153 & 5.21 & -36 & 12 & 58 \\
\hline Left Middle Frontal/Precentral & 8 & 41 & 3.83 & -32 & 12 & 42 \\
\hline Left Posterior Temporal/Parietal & 40 & 1,697 & 6.17 & -34 & -56 & 48 \\
\hline Left Inferior Temporal & 20 & 29 & 3.78 & -54 & -20 & -30 \\
\hline Left Inferior/Middle Temporal & 37 & 38 & 3.52 & -58 & -48 & -10 \\
\hline Left Angular Gyrus & 39 & 40 & 3.96 & 32 & -52 & 28 \\
\hline Bilateral Precuneus & 7 & 589 & 4.46 & -2 & -66 & 44 \\
\hline Left Hippocampus, Cingulum & 30 & 245 & 5.35 & -20 & -42 & 8 \\
\hline Right Hippocampus & & 337 & 5.56 & 38 & -44 & 2 \\
\hline Right Cerebellum & & 310 & 5.55 & 34 & -86 & -38 \\
\hline \multicolumn{7}{|l|}{ (C) Difficult: Opposite condition } \\
\hline Left Inferior/Middle Frontal & 47 & 5,056 & 13.67 & -54 & 22 & -8 \\
\hline Right Inferior/Middle Frontal & 45 & 2,485 & 10.50 & 58 & 28 & 18 \\
\hline Bilateral Medial/Superior Frontal & 8 & 3,274 & 9.57 & -6 & 44 & 44 \\
\hline Left Posterior Temporal/Parietal & 37 & 4,115 & 9.83 & -52 & -36 & -8 \\
\hline Right Middle/Inferior Temporal & 20 & 859 & 5.85 & 56 & -36 & -12 \\
\hline Right Posterior Temporal/Parietal & 39 & 180 & 4.35 & 62 & -58 & 26 \\
\hline Right Parietal & 40 & 55 & 3.49 & 40 & -54 & 42 \\
\hline Bilateral Precuneus & 7 & 623 & 5.20 & -4 & -62 & 40 \\
\hline Left Caudate & & 250 & 5.14 & -6 & -2 & 24 \\
\hline Left Hippocampus & & 168 & 4.53 & -24 & -48 & 8 \\
\hline Left Cerebellum & & 233 & 4.74 & -10 & -86 & -38 \\
\hline Right Cerebellum & & 625 & 8.57 & 30 & -84 & -38 \\
\hline \multicolumn{7}{|l|}{ (D) Difficult-Easy contrast } \\
\hline Superior Medial Frontal & 8 & 1,152 & 7.29 & -4 & 36 & 52 \\
\hline Left Inferior Frontal & 47 & 698 & 6.24 & -52 & 24 & -12 \\
\hline Left Middle Frontal/Inferior Frontal & 8 & 726 & 5.15 & -42 & 6 & 48 \\
\hline Right Inferior/Orbital Frontal & 47 & 221 & 5.19 & 44 & 28 & -14 \\
\hline Right Inferior Frontal & 46 & 106 & 4.58 & 58 & 26 & 26 \\
\hline Right Middle Frontal & 9 & 84 & 4.58 & 42 & 20 & 44 \\
\hline Right Superior Medial Frontal & 6 & 16 & 4.36 & 14 & 28 & 64 \\
\hline Right Inferior Frontal & $45 / 46$ & 13 & 3.97 & 58 & 30 & 10 \\
\hline Left Middle Temporal & $21 / 22$ & 161 & 4.31 & -56 & -36 & -6 \\
\hline Left Striatum & & 45 & 4.12 & -16 & 4 & 10 \\
\hline Left Cerebellum & & 33 & 4.01 & -10 & -90 & -34 \\
\hline Right Cerebellum & & 98 & 4.99 & 16 & -84 & -38 \\
\hline
\end{tabular}

Note. $\quad \mathrm{MNI}=$ Montreal Neurological Institute.

comparisons are listed in Table 4. Bar graphs illustrating means and standard errors for left and right inferior/orbital frontal ROIs are depicted in Figure 3.

\section{Effects of Figurativeness}

Group distribution of activation analyses showed no effects of figurativeness. Neither the contrast of all figurative conditions (averaged across difficulty) minus literal nor the three independent contrasts of each figurative condition minus the literal condition revealed reliable results.

Volumetric analyses of individual subject data, however, did provide evidence for effects of figurativeness. $t$ tests revealed reliably greater activation in the three averaged figurative-literal conditions in the right temporo-parietal ROI, $t(23)=2.15$; the right middle frontal ROI, $t(23)=3.27$; and the superior medial frontal ROI, $t(23)=2.21$. 
Table 4

Analysis of Variance Statistics for ROIs With Reliable Effects, With Mean Number of Voxels (Standard Error in Parentheses) and Follow-Up T Statistics for Each of the Three Difficulty Conditions

\begin{tabular}{|c|c|c|c|c|c|c|c|c|}
\hline ROI & $F(2,22)$ & $M S E$ & $\begin{array}{l}\text { Mean Support } \\
\text { Context (1) }\end{array}$ & $\begin{array}{l}\text { Mean Neutral } \\
\text { Context (2) }\end{array}$ & $\begin{array}{l}\text { Mean Opposite } \\
\text { Context (3) }\end{array}$ & $\mathrm{T}(1-2)$ & $\mathrm{T}(1-3)$ & $\mathrm{T}(2-3)$ \\
\hline LH Inferior/Orbital Frontal & $12.78^{* *}$ & 36,289 & $102(28)$ & $207(43)$ & $367(44)$ & -1.98 & $-5.16^{* *}$ & $-2.67^{*}$ \\
\hline LH Inferior/Middle Frontal & $4.96^{*}$ & 56,249 & $173(59)$ & $237(59)$ & $382(59)$ & -0.93 & $-3.18^{* *}$ & -2.05 \\
\hline LH Superior Frontal & $5.23^{*}$ & 13,264 & $48(22)$ & $72(25)$ & $153(36)$ & -0.73 & $-3.19^{* * *}$ & $-2.42^{*}$ \\
\hline Inferior Medial Frontal & $5.25^{*}$ & 18,512 & $36(19)$ & $81(29)$ & $157(32)$ & -1.26 & $-3.28^{* *}$ & -1.71 \\
\hline RH Inferior/Orbital Frontal & $5.14^{*}$ & 18,610 & $47(17)$ & $76(27)$ & 187 (17) & -0.93 & $-3.27^{* *}$ & $-2.6^{*}$ \\
\hline RH Inferior/Middle Frontal & $3.93^{*}$ & 57,751 & $137(63)$ & $67(25)$ & $253(60)$ & 0.99 & -1.59 & $-2.86^{* * *}$ \\
\hline
\end{tabular}

Note. $\quad$ ROI $=$ region of interest.

${ }^{*} p<.05 .{ }^{* *} p<.01$.

\section{Effects of Individual Vocabulary Size}

Individuals with higher vocabulary scores had less, or more efficient, activation in each of the three figurative utterance conditions than did individuals with lower vocabulary scores. The resulting negative correlations between activation levels and vocabulary percentile were consistently observed in the right inferior frontal gyrus. In addition, during the supporting context condition, increased activation in less-experienced readers was observed in a number of regions including left inferior and middle frontal gyri, the right middle frontal gyrus, the right parietal lobe, the cingulum, the precuneus, and the striatum. Activation maps depicting the

\section{Effects of Contextual Difficulty on Frontal Activation Volumes}
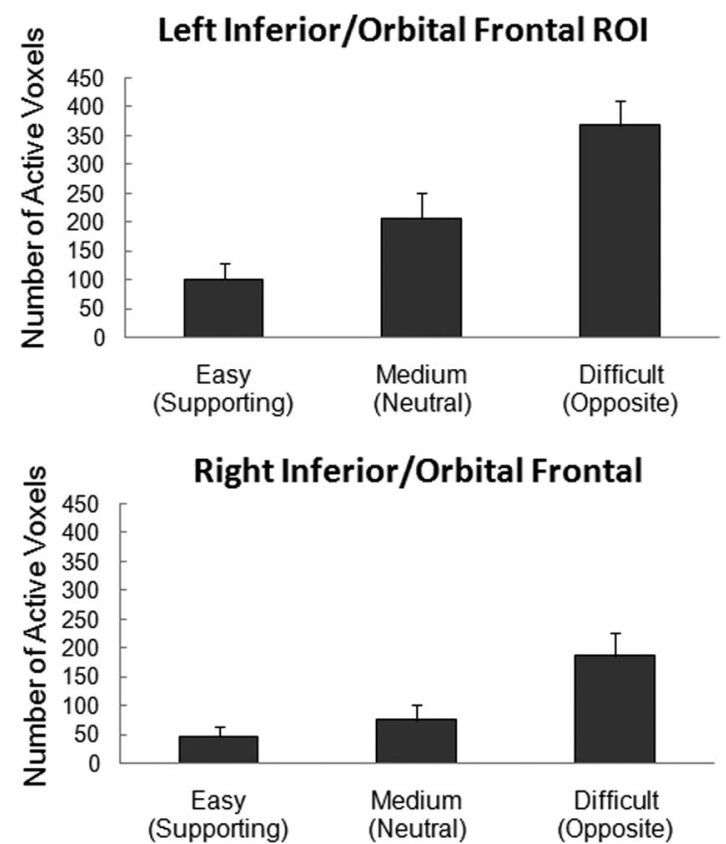

Figure 3. Bar graphs depicting the mean number of voxels active (error bars depict standard error of the mean) in the volumetric analyses of the three context conditions for the left inferior/orbital frontal region of interest (ROI; top) and the right inferior/orbital frontal ROI (bottom). regions where activation was reliably negatively correlated with vocabulary size during supporting (in red), neutral (in yellow), and ironic (in blue) context conditions and their overlap in the right inferior frontal gyrus are depicted in Figure 4. Positive correlations between vocabulary size and activation were observed in one region of the right occipital lobe during the neutral context condition only.

Individuals with higher vocabulary scores also showed a greater processing benefit from supporting context than did individuals with lower vocabulary. This resulted in a larger decrease in activation in working memory and conflict monitoring regions in higher vocabulary individuals when supporting context was presented. Thus, when the contrast of opposite minus supporting context conditions was entered as the dependent variable, vocabulary size was reliably positively correlated with contrast values in bilateral middle frontal gyri and in the cingulate. Similarly, when the contrast of neutral minus supporting contexts was entered as the dependent variable, vocabulary size was reliably positively correlated with contrast values in the left middle frontal gyrus. MNI coordinates, Brodmann's areas, and peak T values of clusters of activation with reliable correlations between vocabulary size in each of the three context conditions, and for the contrasts of opposite-supporting contexts and neutral-supporting contexts, are listed Table 5.

\section{Effects of Individual Working Memory Capacity}

Individuals with higher working memory capacities also had more efficient patterns of activation than did individuals with lower working memory capacities. The resulting negative correlations between activation and reading span were observed in executive function (cingulate) and memory (precuneus) regions in the supporting context conditions but were observed only in visual processing regions (e.g., right fusiform gyrus) during the difficult, opposite context conditions. No correlations were observed between reading span and activation in the neutral context condition.

Individuals with higher working memory capacity also showed a greater benefit from supporting context than did individuals with lower capacity. When the contrast of opposite-supporting contexts was entered as the dependent variable, reading span was reliably positively correlated with activation in memory regions including the right hippocampus and left precuneus. No reliable correlations were observed between reading span and activation in the contrast 


\section{Increased Right Hemisphere Activation in Less-Skilled Readers during Supporting(red), Neutral (yellow), and Opposite (blue) Context Conditions}

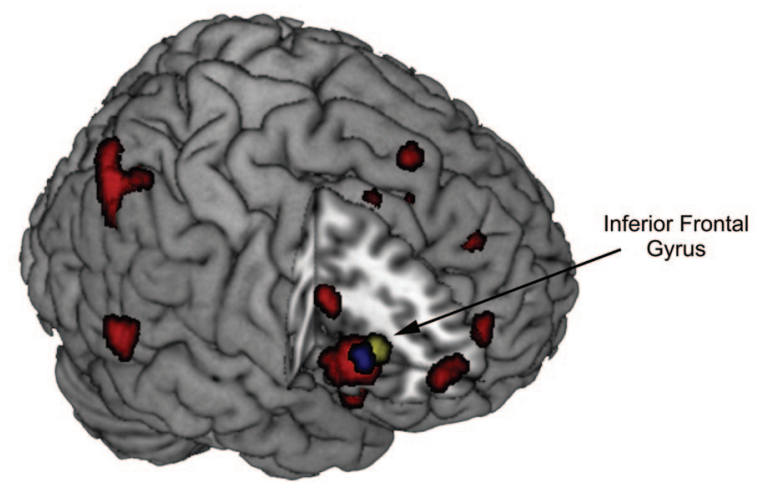

Figure 4. Activation map showing regions of reliable negative correlations between individual vocabulary size and activation during the easy (red), neutral (yellow) and difficult (blue) context conditions.

of neutral minus supporting context conditions. MNI coordinates, Brodmann's areas, and peak T values of clusters of activation with reliable correlations between reading span and activation are listed Table 6.

\section{Discussion}

\section{Information-Processing Demands and RH Activation During Metaphor Comprehension}

The results from this experiment support the view that the RH is dynamically recruited as a function of the processing demands of a language task. We provide converging evidence that the $\mathrm{RH}$ becomes increasingly involved in metaphor comprehension when the processing demands of the task increase. First, group distribution of activation analyses showed increased activation in the right inferior and middle frontal gyri only during the most difficult condition, namely, when preceding context biased an opposite, or ironic interpretation of the utterance (as illustrated in Figure 2 and Table 3). Second, volumetric analyses of activation in the right inferior frontal gyrus showed linear increases in activation with difficulty (as shown in Figure 3). Finally, decreased individual reading skill (which is presumably related to increased experienced processing demands) was also associated with increased activation in both the right inferior frontal gyrus and in right frontopolar regions (as depicted in Figure 4 and Table 5).

These findings are consistent with the dynamic spillover account of RH involvement in discourse comprehension, and with research on analogical reasoning suggesting that the RLPFC is sensitive to processing demands (e.g., Bunge et al., 2005, 2009). The results are also consistent with previous research showing increased RH involvement with increased task demands (e.g., Just et al., 1996; Yang et al., 2009) and with decreased individual reading skill (e.g., Prat, Keller, \& Just, 2007; Prat, Long, \& Baynes, 2007; Prat et al., 2010). Another possible account for the increased RH involvement with increasing processing demands is that it reflects a greater need for more general cognitive processes, such as response selection and/or inhibition (e.g., Dodds et al., 2010). The condition in which RH inferior frontal activation was observed in the group data was also the condition in which the dominant interpretation (sincere metaphor) needed to be suppressed to accurately comprehend the critical utterances as an ironic statement. One might also argue that increased $\mathrm{RH}$ inferior frontal activation in less-skilled readers reflects a greater need for response selection that arises from inefficient suppression of inappropriate responses (e.g., Gernsbacher, Keysar, Robertson, \& Werner, 2001).

The results cannot be entirely explained by the coarse-coding theory. Although the semantic relatedness of the critical utterance target and vehicle did not vary across contexts in this study, one might argue that the search for meaning here was driven not only by the semantic relatedness of the items in the critical utterance but also by the need to integrate the overlapping semantic relations in a meaningful way with the existing content. Coarse coding does not readily explain the observed effect of skill differences on $\mathrm{RH}$ activation.

It is worth noting that not all areas of the $\mathrm{RH}$ were sensitive to the difficulty manipulation. In particular, volumetric analyses of the right temporo-parietal and posterior superior medial frontal regions suggest that they were sensitive to figurativeness and not to difficulty manipulations. Both of these regions exhibited increased activation across all figurative conditions compared with the literal condition. Thus, these regions seemed sensitive to figurativeness, rather than more general processing demands. Based on previous research, it is plausible that these areas are responding to the increased social processing demands of the three figurative conditions. Specifically, in each of the figurative conditions, insights into the intentions (or theory of mind) of the protagonist were relevant for comprehending the critical utterance. In the literal condition, the protagonist's mental processes were less relevant for interpreting the meaning of the critical utterance, as he or she was simply stating a literal truth. Because figurativeness and theory of mind regions covaried, it is not possible to determine which factor primarily contributed to increased activation in these regions. However, the opposite context condition, which arguably is the most demanding of theory of mind processing (the reader must understand that the speaker means something different than 
Table 5

Significant Correlations Between Activation Contrasts and Vocabulary Size

\begin{tabular}{|c|c|c|c|c|c|c|}
\hline \multirow[b]{2}{*}{ Cortical region } & \multirow[b]{2}{*}{ Peak Brodmann's area } & \multirow[b]{2}{*}{ Cluster size } & \multirow[b]{2}{*}{ Peak $\mathrm{T}$ value } & \multicolumn{3}{|c|}{ MNI coordinates } \\
\hline & & & & $x$ & $y$ & $z$ \\
\hline \multicolumn{7}{|l|}{ (A) Easy: Supporting condition } \\
\hline \multicolumn{7}{|l|}{ Negative correlation with vocabulary size } \\
\hline Right Inferior Frontal/Insula & 45 & 325 & 5.16 & 50 & 24 & 10 \\
\hline Right Inferior Frontal/Insula & 13 & 31 & 3.87 & 38 & 8 & 20 \\
\hline Right Frontopolar & 10 & 15 & 4.01 & 36 & 60 & 20 \\
\hline Right Frontopolar & 10 & 27 & 3.77 & 46 & 50 & 6 \\
\hline Right Middle/Superior Frontal & 8 & 16 & 3.84 & 20 & 16 & 48 \\
\hline Right Medial Frontal/ Anterior Cingulum & 9 & 33 & 4.18 & 8 & 44 & 32 \\
\hline Right Superior Medial Frontal/Cingulum & 6 & 14 & 3.66 & 10 & 28 & 42 \\
\hline Right Medial Frontal & 6 & 14 & 3.95 & 10 & 26 & 62 \\
\hline Right Cingulum & 23 & 54 & 3.99 & 10 & -44 & 22 \\
\hline Right Middle/Superior Temporal & 22 & 49 & 4.51 & 64 & -56 & 10 \\
\hline Right Superior Parietal/Occipital & 39 & 503 & 5.08 & 34 & -64 & 40 \\
\hline Left Inferior Frontal & 44 & 132 & 5.07 & -46 & 18 & 10 \\
\hline Left Inferior Frontal/Insula & 47 & 280 & 5.18 & -36 & 28 & 2 \\
\hline Left Middle Frontal & 9 & 18 & 4.18 & -30 & 20 & 30 \\
\hline Left Precuneus/Cuneus & 7 & 91 & 4.37 & -8 & -78 & 38 \\
\hline Left Thalamus/Basal Ganglia & & 135 & 5.24 & -12 & -6 & 4 \\
\hline Right Basal Ganglia & & 414 & 4.97 & 8 & 0 & 0 \\
\hline Bilateral Thalamus & & 16 & 3.70 & 0 & -12 & 8 \\
\hline Left Cerebellum & & 21 & 3.78 & -12 & -74 & -36 \\
\hline \multicolumn{7}{|l|}{ (B) Medium: Neutral condition } \\
\hline \multicolumn{7}{|l|}{ Negative correlation with vocabulary size } \\
\hline Right Inferior Frontal & 13 & 22 & 4.07 & 42 & 26 & 8 \\
\hline \multicolumn{7}{|l|}{ Positive correlation with vocabulary size } \\
\hline Right Occipital & $18 / 19$ & 55 & 5.33 & 24 & -92 & 0 \\
\hline \multicolumn{7}{|l|}{ (C) Difficult: Opposite condition } \\
\hline \multicolumn{7}{|l|}{ Negative correlation with vocabulary size } \\
\hline Right Inferior Frontal & 45 & 17 & 4.04 & 50 & 24 & 10 \\
\hline Left Parietal & 40 & 26 & 4.83 & -62 & -52 & 32 \\
\hline \multicolumn{7}{|l|}{ (D) Neutral: Supporting contrast } \\
\hline \multicolumn{7}{|l|}{ Positive correlation with vocabulary size } \\
\hline Left Middle/Inferior Frontal & 9 & 74 & 4.83 & -32 & 26 & 26 \\
\hline Left Middle/Superior Frontal & 8 & 13 & 3.84 & -26 & 34 & 38 \\
\hline Right Middle Occipital & 18 & 19 & 3.91 & 36 & -70 & 26 \\
\hline \multicolumn{7}{|l|}{ (E) Opposite: Supporting contrast } \\
\hline \multicolumn{7}{|l|}{ Positive correlation with vocabulary size } \\
\hline Right Middle Frontal & 45 & 51 & 4.35 & 46 & 46 & 24 \\
\hline Left Middle/Inferior Frontal & 9 & 15 & 3.79 & -34 & 24 & 26 \\
\hline Posterior Cingulum/Precuneus & & 49 & 4.44 & 10 & -44 & 24 \\
\hline Anterior Cingulum & & 19 & 4.01 & -4 & 6 & 26 \\
\hline
\end{tabular}

Note. $\quad \mathrm{MNI}=$ Montreal Neurological Institute.

what he or she is saying), also resulted in the most activation in the right temporo-parietal regions in the distribution of activation maps. It is worth noting that increased activation in the superior medial frontal and right temporo-parietal regions was not observed at the group level in neutral or supporting context conditions. This may be due to increased individual differences in these regions in the supporting context condition (as seen in Figure 4 and listed in Table 5), or to the fact that the precise locations of activation are more variable across participants in the RH (e.g., Prat et al., 2010) and thus don't reach group-level significance on a voxel-by-voxel level. Taken together, these results provide evidence that rather than having a unique, consistent role in metaphor comprehension processes, the RH's involvement in metaphor is related to more general, information processing demands placed by the task.

\section{Comparing Metaphor and Analogy in the Brain}

The results of this experiment highlight the overlapping neural and computational components of metaphor comprehension and analogical reasoning. In the easiest, supported metaphor comprehension condition, readers were given a context indicating the relevant semantic information necessary for relational reasoning about the topic and vehicle of the critical, metaphoric utterance. In this condition, increased activation was observed only in a select, inferior region of the LLPFC. This region was highly overlapping with the regions reported in investigations of verbal analogical reasoning (e.g., Bunge et al., 2005; Volle et al., 2010; Wendelken et al., 2008) and was consistently active in each of the metaphoric conditions. As the processing demands of metaphor comprehension increased (by decreasing the support of context sentences), 
Table 6

Significant Correlations Between Activation Contrasts and Individual Reading Span

\begin{tabular}{|c|c|c|c|c|c|c|}
\hline \multirow[b]{2}{*}{ Cortical region } & \multirow[b]{2}{*}{ Peak Brodmann's area } & \multirow[b]{2}{*}{ Cluster size } & \multirow[b]{2}{*}{ Peak T value } & \multicolumn{3}{|c|}{ MNI coordinates } \\
\hline & & & & $x$ & $y$ & $z$ \\
\hline \multicolumn{7}{|l|}{ (A) Supporting condition } \\
\hline \multicolumn{7}{|l|}{ Negative correlation with reading span } \\
\hline Anterior Cingulum/Medial Orbital Frontal & 32 & 30 & 4.68 & 4 & 30 & -8 \\
\hline Middle Cingulum & 24 & 25 & 4.30 & -6 & 2 & 34 \\
\hline Left Middle Occipital & $18 / 19$ & 19 & 3.99 & -44 & -88 & 0 \\
\hline \multicolumn{7}{|l|}{ (B) Opposite condition } \\
\hline \multicolumn{7}{|l|}{ Negative correlation with reading span } \\
\hline Right Fusiform Gyrus & 19 & 32 & 5.30 & 28 & -74 & -2 \\
\hline Right Superior Occipital & 19 & 13 & 3.72 & 26 & -84 & 42 \\
\hline Left Occipital & 19 & 36 & 4.66 & -46 & -86 & 6 \\
\hline \multicolumn{7}{|l|}{ (C) Opposite: Supporting contrast } \\
\hline \multicolumn{7}{|l|}{ Positive correlation with reading span } \\
\hline Right Hippocampus & & 49 & 4.51 & 36 & -34 & -2 \\
\hline Left Precuneus & 7 & 13 & 3.98 & -12 & -56 & 44 \\
\hline
\end{tabular}

Note. $\quad \mathrm{MNI}=$ Montreal Neurological Institute.

areas typically associated with working memory processes (e.g., middle frontal gyrus and parietal lobe) and areas involved in response selection (e.g., right inferior frontal gyrus) became increasingly involved. Activation of the left frontopolar cortex (BA 10) was only observed in the neutral condition, in which context sentences did not provide any relevant information for reasoning about the relations between the topic and vehicle of the metaphoric utterance. Thus, it is possible that this condition required the most relational processing at the critical utterance. These findings are consistent with analogical reasoning literature suggestions that LLPFC is necessary for relational reasoning, whereas the RLPFC is sensitive to the general processing demands of the task.

\section{The Neural Bases of Individual Differences in Metaphor Comprehension}

Individual differences in cognitive capabilities were reflected by differential recruitment and modulation of a network of higher level cognitive areas during metaphor comprehension. Consistent with our previous research, we found that increased vocabulary size, a measure that is highly correlated with increased reading experience (e.g., Stanovich \& West, 1989; Stanovich, West, \& Harrison, 1995), is related to increased neural efficiency during a variety of reading comprehension tasks (e.g., Prat \& Just, 2011; Prat et al., 2010). Although previous research has shown that individual differences in working memory capacity are also related to differences in neural efficiency (e.g., Prat, Keller, \& Just, 2007), the current findings are consistent with more recent research suggesting that when indices of reading experience, such as vocabulary size, and indices of more general cognitive abilities, such as working memory capacity, are used as independent regressors, vocabulary size is more strongly related to neural efficiency (Prat \& Just, 2011). Such increases in efficiency tend to be most apparent in the RH homologues of LH language regions (e.g., Prat \& Just, 2011; Prat et al., 2010). As described above, the increased RH activation in readers with lower vocabulary is readily explained by RH spillover. According to the dynamic spillover hypothesis, this increased $\mathrm{RH}$ activation reflects a higher ratio of processing demands to neural resource availability in individuals with lower vocabulary. Thus, the dominant LH of lower capacity individuals is more quickly depleted of neural resources, resulting in greater recruitment of RH processing reserves.

Although increased RH activation in individuals with lower vocabulary was observed under all processing demands, individual differences in working memory and cognitive control regions were primarily observed in the supporting context condition. Also, despite the fact that the supporting context condition was the least cognitively demanding, it was the condition in which individuals were most likely to integrate the relevant relational information mentioned in the context to their semantic representation of the mappings between the topic and vehicle in the critical utterance. Thus, increased activation in bilateral middle frontal gyri in individuals with lower vocabulary in this condition may reflect an increased difficulty maintaining or retrieving the relevant contextual information in working memory. Additionally, increased anterior cingulate activation observed both in individuals with lower vocabulary and lower capacity in this condition may reflect increased attentional demands or conflict monitoring. Individual differences in memory and executive function regions were also observed when investigating the facilitative effects of supporting context on activation. Specifically, individuals with higher vocabulary showed a greater decrease in activation in bilateral middle frontal and cingulate regions when supporting context was presented (vs. neutral or opposite context), and individuals with higher working memory capacity showed a greater decrease in activation in episodic memory-retrieval regions when supporting context was presented (vs. opposite context). These results may indicate that individuals with higher working memory capacity more readily maintain relevant semantic information during the context phase, whereas individuals with lower working memory capacity resort to episodic memory retrieval at a 
later stage, when the information is needed to comprehend the critical utterance.

\section{Summary}

Metaphor comprehension, like relational reasoning and other high-level cognitive tasks, relies on complex interactions between task characteristics and individual cognitive abilities. To accurately understand metaphor comprehension, one must be able to independently characterize the variables related to the metaphor processing demands (e.g., semantic relatedness, difficulty, and familiarity). One must also understand the facets of individual cognitive profiles (e.g., reading experience and working memory capacity) and how they interact with task variables to generate experienced processing demands. Results from this experiment highlight the importance of understanding metaphor comprehension in terms of dynamic information-processing characteristics that generalize not only to other, literal and figurative types of discourse comprehension but also to reasoning and thinking in general.

\section{References}

Aron, A. R., Robbins, T. W., \& Poldrack, R. A. (2004). Inhibition and the right inferior frontal cortex. Trends in Cognitive Sciences, 8, 170-177. doi:10.1016/j.tics.2004.02.010

Beeman, M., \& Chiarello, C. (Eds.). (1998). Right hemisphere language comprehension: Perspectives from cognitive neuroscience. Mahwah, NJ: Erlbaum.

Beeman, M., Friedman, R. B., Grafman, J., Perez, E., Diamond, S., \& Lindsay, M. B. (1994). Summation priming and coarse semantic coding in the right hemisphere. Journal of Cognitive Neuroscience, 6, 26-45. doi:10.1162/jocn.1994.6.1.26

Blasko, D. G. (1999). Only the tip of the iceberg: Who understands what about metaphor? Journal of Pragmatics, 31, 1675-1683. doi:10.1016/ S0378-2166(99)00009-0

Bowdle, B. F., \& Gentner, D. (2005). The career of metaphor. Psychological Review, 112, 193-216. doi:10.1037/0033-295X.112.1.193

Brown, J. A., Fishco, V. V., \& Hanna, G. (1993). Nelson-Denny Reading Test: Manual for scoring and interpretation, Forms $G \& H$. Rolling Meadows, IL: Riverside.

Brownell, H. H., Simpson, T. L., Bihrle, A. M., Potter, H. H., \& Gardner, H. (1990). Appreciation of metaphoric alternative word meanings by left and right brain-damaged patients. Neuropsychologia, 28, 375-383. doi: 10.1016/0028-3932(90)90063-T

Bunge, S. A., Helskog, E. H., \& Wendelken, C. (2009). Left, but not right, rostrolateral prefrontal cortex meets a stringent test of the relational integration hypothesis. NeuroImage, 46, 338-342. doi:10.1016/ j.neuroimage.2009.01.064

Bunge, S., Wendelken, C., Badre, D., \& Wagner, A. (2005). Analogical reasoning and prefrontal cortex: Evidence for separable retrieval and integration mechanisms. Cerebral Cortex, 15, 239-249. doi:10.1093/ cercor/bhh126

Cho, S., Moody, T., Fernandino, L., Mumford, J., Poldrack, R., Cannon, T., ... Holyoak, K. (2010). Common and dissociable prefrontal loci associated with component mechanisms of analogical reasoning. Cerebral Cortex, 20, 524-533. doi:10.1093/cercor/bhp121

Daneman, M., \& Carpenter, P. A. (1980). Individual differences in working memory and reading. Journal of Verbal Learning and Verbal Behavior, 19, 450-466. doi:10.1016/S0022-5371(80)90312-6

Dodds, C. M., Morein-Zamir, S., \& Robbins, T. W. (2010). Dissociating inhibition, attention, and response control in the frontoparietal network using functional magnetic resonance imaging. Cerebral Cortex, 21 , 1155-1165. doi:10.1093/cercor/bhq187

Eviatar, Z., \& Just, M. (2006). Brain correlates of discourse processing: An fMRI investigation of irony and conventional metaphor comprehension. Neuropsychologia, 44, 2348-2359. doi:10.1016/j.neuropsychologia .2006.05.007

Ferstl, E. C., Neumann, J., Bogler, C., \& von Cramon, D. Y. (2008). The extended language network: A meta-analysis of neuroimaging studies on text comprehension. Human Brain Mapping, 29, 581-593. doi:10.1002/ hbm. 20422

Fritson, K. J., Holmes, A. P., Worsley, K. J., Poline, J.-B, Frith, C. D., \& Frackowiak, R. S. J. (1995). Statistical parametric maps in functional imaging: A general linear approach. Human Brain Mapping, 2, 189 210

Geake, J., \& Hansen, P. (2010). Functional neural correlates of fluid and crystallized analogizing. NeuroImage, 49, 3489-3497. doi:10.1016/ j.neuroimage.2009.09.008

Genovese, C. R., Lazar, N. A., \& Nichols, T. (2002). Thresholding of statistical maps in functional neuroimaging using the false discovery rate. NeuroImage, 15, 870-878. doi:10.1006/nimg.2001.1037

Gentner, D. (1983). Structure-mapping: A theoretical framework for analogy. Cognitive Science, 7, 155-170. doi:10.1207/s15516709cog0702_3

Gentner, D., Bowdle, B. F., Wolff, P., \& Boronat, C. (2001). Metaphor is like analogy. In D. Gentner, K. J. Holyoak, \& B. N. Kokinov (Eds.), The analogical mind: Perspectives from cognitive science (pp. 199-253). Cambridge, MA: MIT Press.

Gentner, D., \& Clement, C. (1988). Evidence for relational selectivity in the interpretation of analogy and metaphor. In Bower, G. H. (Ed.), The psychology of learning and motivation: Advances in research and theory (Vol. 22, pp. 307-358). San Diego, CA: Academic Press.

Gentner, D., \& Holyoak, K. J. (1997). Reasoning and learning by analogy. American Psychologist, 52, 32-34. doi:10.1037/0003-066X.52.1.32

Gernsbacher, M. A., Keysar, B., Robertson, R. R. W., \& Werner, N. K. (2001). The role of suppression and enhancement in understanding metaphors. Journal of Memory and Language, 45, 433-450. doi: 10.1006/jmla.2000.2782

Giora, R. (1999). On the priority of salient meanings: Studies of literal and figurative language. Journal of Pragmatics, 31, 919-929. doi:10.1016/ S0378-2166(98)00100-3

Giora, R. (2007). Is metaphor special? Brain and Language, 100, 111-114. doi:10.1016/j.bandl.2006.08.001

Glucksberg, S. (2003). The psycholinguistics of metaphor. Trends in Cognitive Sciences, 7, 92-96. doi:10.1016/S1364-6613(02)00040-2

Goghari, V. M., \& MacDonald, A. W. (2009). The neural basis of cognitive control: Response selection and inhibition. Brain Cognition, 71, 72-83. doi:10.1016/j.bandc.2009.04.004

Green, A. E., Fugelsang, J. A., Kraemer, D. J., Shamosh, N. A., \& Dunbar, K. N. (2006). Frontopolar cortex mediates abstract integration in analogy. Brain Research, 1096, 125-137. doi:10.1016/j.brainres .2006 .04 .024

Hatta, T. (1982). Differential effects of concurrent letters on visual field differences in letter recognition. International Journal of Neuroscience, 17, 129-134. doi:10.3109/00207458208985099

Haun, F. (1981). Functionally lateralised information processing and its allocation to response: Inferences from three measures of letter identification performance following lateralised visual input. Quarterly Journal of Experimental Psychology A, 33, 275-294. doi:10.1080/ 14640748108400793

Holyoak, K. J., \& Thagard, P. (1997). The analogical mind. American Psychologist, 52, 35-44. doi:10.1037/0003-066X.52.1.35

Jung-Beeman, M. (2005). Bilateral brain processes for comprehending natural language. Trends in Cognitive Sciences, 9, 512-518. doi: 10.1016/j.tics.2005.09.009

Just, M. A., Carpenter, P. A., Keller, T. A., Eddy, W. F., \& Thulborn, K. R. 
(1996). Brain activation modulated by sentence comprehension. Science, 274, 114-116. doi:10.1126/science.274.5284.114

Kazmerski, V. A., Blasko, D. G., \& Dessalegn, B. G. (2003). ERP and behavioral evidence of individual differences in metaphor comprehension. Memory \& Cognition, 31, 673-689. doi:10.3758/BF03196107

Krawczyk, D., McClelland, M., Donovan, C., Tillman, G., \& Maguire, M. (2010). An fMRI investigation of cognitive stages in reasoning by analogy. Brain Research, 1342, 63-73. doi:10.1016/j.brainres.2010.04.039

Lee, S. S., \& Dapretto, M. (2006). Metaphorical vs. literal word meanings: fMRI evidence against a selective role of the right hemisphere. NeuroImage, 29, 536-544. doi:10.1016/j.neuroimage.2005.08.003

Mashal, N., \& Faust, M. (2009). Conventionalisation of novel metaphors: A shift in hemispheric asymmetry. Laterality, 14, 573-589. doi:10.1080/ 13576500902734645

Mashal, N., Faust, M., Hendler, T., \& Jung-Beeman, M. (2007). An fMRI investigation of the neural correlates underlying the processing of novel metaphoric expressions. Brain and Language, 100, 115-126. doi: 10.1016/j.bandl.2005.10.005

Mason, R. A., \& Just, M. A. (2009). The role of the theory-of-mind cortical network in the comprehension of narratives. Language and Linguistics Compass, 3, 157-174. doi:10.1111/j.1749-818X.2008.00122.x

Mason, R. A., \& Just, M. A. (2011). Differentiable cortical networks for inferences concerning people's intentions versus physical causality. $\mathrm{Hu}$ man Brain Mapping, 32, 313-329. doi:10.1002/hbm.21021

Mitchell, J. P. (2008). Activity in right temporo-parietal junction is not selective for theory-of-mind. Cerebral Cortex, 18, 262-271. doi: 10.1093/cercor/bhm051

Mitchell, R. L., \& Ross, E. D. (2008). fMRI evidence for the effect of verbal complexity on lateralisation of the neural response associated with decoding prosodic emotion. Neuropsychologia, 46, 2880-2887. doi:10.1016/j.neuropsychologia.2008.05.024

Monetta, L., Ouellet-Plamondon, C., \& Joanette, Y. (2006). Simulating the pattern of right-hemisphere-damaged patients for the processing of the alternative metaphorical meanings of words: Evidence in favor of a cognitive resources hypothesis. Brain and Language, 96, 171-177. doi:10.1016/j.bandl.2004.10.014

Oldfield, R. C. (1971). The assessment and analysis of handedness: The Edinburgh Inventory. Neuropsychologia, 9, 97-113. doi:10.1016/00283932(71)90067-4

Prat, C. S., \& Just, M. A. (2008). Brain bases of individual differences in cognition. Psychological Science Agenda, 22.

Prat, C. S., \& Just, M. A. (2011). Exploring the cortical dynamics underpinning individual differences in sentence comprehension. Cerebral Cortex, 21, 1747-1760.

Prat, C. S., Keller, T. A., \& Just, M. A. (2007). Individual differences in sentence comprehension: An fMRI investigation of syntactic and lexical processing demands. Journal of Cognitive Neuroscience, 19, 19501963. doi:10.1162/jocn.2007.19.12.1950

Prat, C. S., Long, D. L., \& Baynes, K. (2007). The representation of discourse in the two hemispheres: An individual differences investigation. Brain and Language, 100, 283-294. doi:10.1016/j.bandl.2006.11.002

Prat, C. S., Mason, R. A., \& Just, M. A. (2010, June). Right hemisphere contributions to reading: A multi-experiment individual differences investigation. Paper presented at the Organization for Human Brain Mapping, Barcelona, Spain.

Prat, C. S., Mason, R. A., \& Just, M. A. (2011). Individual differences in the neural basis of causal inferencing. Brain and Language, 116, 1-13. doi:10.1016/j.bandl.2010.08.004

Rapp, A., Leube, D., Erb, M., Grodd, W., \& Kircher, T. (2004). Neural correlates of metaphor processing. Brain Research Cognitive Brain Research, 20, 395-402. doi:10.1016/j.cogbrainres.2004.03.017

Rapp, A., Leube, D., Erb, M., Grodd, W., \& Kircher, T. (2007). Laterality in metaphor processing: Lack of evidence from functional magnetic resonance imaging for the right hemisphere theory. Brain and Language, 100, 142-149. doi:10.1016/j.bandl.2006.04.004

Rinaldi, M. C., Marangolo, P., \& Baldassarri, F. (2004). Metaphor comprehension in right brain-damaged patients with visuo-verbal and verbal material: A dissociation (re)considered. Cortex, 40, 479-490. doi: 10.1016/S0010-9452(08)70141-2

Saxe, R., \& Kanwisher, N. (2003). People thinking about people: The role of the temporo-parietal junction in "theory of mind." NeuroImage, 19, 1835-1842. doi:10.1016/S1053-8119(03)00230-1

Saxe, R., \& Powell, L. J. (2006). It's the thought that counts: Specific brain regions for one component of theory of mind. Psychological Science, 17, 692-699. doi:10.1111/j.1467-9280.2006.01768.x

Saxe, R., \& Wexler, A. (2005). Making sense of another mind: The role of the right temporoparietal junction. Neuropsychologia, 43, 1391-1399. doi:10.1016/j.neuropsychologia.2005.02.013

Schmidt, G., \& Seger, C. (2009). Neural correlates of metaphor processing: The roles of figurativeness, familiarity and difficulty. Brain and Cognition, 71, 375-386. doi:10.1016/j.bandc.2009.06.001

Shibata, M., Abe, J., Terao, A., \& Miyamoto, T. (2007). Neural mechanisms involved in the comprehension of metaphoric and literal sentences: An fMRI study. Brain Research, 1166, 92-102. doi:10.1016/ j.brainres.2007.06.040

Stanovich, K. E., \& West, R. F. (1989). Exposure to print and orthographic processing. Reading Research Quarterly, 24, 402-433. doi:10.2307/747605

Stanovich, K. E., West, R. F., \& Harrison, M. R. (1995). Knowledge growth and maintenance across the life span: The role of print exposure. Developmental Psychology, 31, 811-826. doi:10.1037/0012-1649.31.5.811

Sternberg, R. J. (1977). Intelligence, information processing, and analogical reasoning: The componential analysis of human abilities. Hillsdale, NJ: Erlbaum.

Sternberg, R. J., \& Nigro, G. (1983). Interaction and analogy in the comprehension and appreciation of metaphors. Quarterly Journal of Experimental Psychology A: Human Experimental Psychology, 35, 1738. doi:10.1080/14640748308402114

Stringaris, A., Medford, N., Giampietro, V., Brammer, M., \& David, A. (2007). Deriving meaning: Distinct neural mechanisms for metaphoric, literal, and non-meaningful sentences. Brain and Language, 100, 150162. doi:10.1016/j.bandl.2005.08.001

Stringaris, A., Medford, N., Giora, R., Giampietro, V., Brammer, M., \& David, A. (2006). How metaphors influence semantic relatedness judgments: The role of the right frontal cortex. Neurolmage, 33, 784-793. doi:10.1016/j.neuroimage.2006.06.057

Trick, L., \& Katz, A. (1986). The domain interaction approach to metaphor processing: Relating individual differences and metaphor characteristics. Metaphor and Symbolic Activity, 1, 185-213. doi:10.1207/s15327868ms0103_3

Volle, E., Gilbert, S., Benoit, R., \& Burgess, P. (2010). Specialization of the rostral prefrontal cortex for distinct analogy processes. Cerebral Cortex, 20, 2647-2659. doi:10.1093/cercor/bhq012

Wendelken, C., Nakhabenko, D., Donohue, S., Carter, C., \& Bunge, S. (2008). "Brain is to thought as stomach is to?" Investigating the role of rostrolateral prefrontal cortex in relational reasoning. Journal of Cognitive Neuroscience, 20, 682-693. doi:10.1162/jocn.2008.20055

Wright, S., Matlen, B., Baym, C., Ferrer, E., \& Bunge, S. (2007). Neural correlates of fluid reasoning in children and adults. Frontiers in Human Neuroscience, 1.

Yang, F., Edens, J., Simpson, C., \& Krawczyk, D. (2009). Differences in task demands influence the hemispheric lateralization and neural correlates of metaphor. Brain and Language, 111, 114-124. doi:10.1016/ j.bandl.2009.08.006

Zinken, J. (2007). Discourse metaphors: The link between figurative language and habitual analogies. Cognitive Linguistics, 18, 445-466. doi: 10.1515/COG.2007.024.

Received December 1, 2010

Revision received July 21, 2011 Accepted July 23, 2011 